ELECTRONIC RESEARCH ANNOUNCEMENTS OF THE AMERICAN MATHEMATICAL SOCIETY

Volume 12, Pages 71-86 (July 7, 2006)

S $1079-6762(06) 00163-6$

\title{
DENSITY, OVERCOMPLETENESS, AND LOCALIZATION OF FRAMES
}

\author{
RADU BALAN, PETER G. CASAZZA, CHRISTOPHER HEIL, AND ZEPH LANDAU
}

(Communicated by Guido Weiss)

\begin{abstract}
This work presents a quantitative framework for describing the overcompleteness of a large class of frames. It introduces notions of localization and approximation between two frames $\mathcal{F}=\left\{f_{i}\right\}_{i \in I}$ and $\mathcal{E}=\left\{e_{j}\right\}_{j \in G}(G$ a discrete abelian group), relating the decay of the expansion of the elements of $\mathcal{F}$ in terms of the elements of $\mathcal{E}$ via a map $a: I \rightarrow G$. A fundamental set of equalities are shown between three seemingly unrelated quantities: the relative measure of $\mathcal{F}$, the relative measure of $\mathcal{E}$ - both of which are determined by certain averages of inner products of frame elements with their corresponding dual frame elements - and the density of the set $a(I)$ in $G$. Fundamental new results are obtained on the excess and overcompleteness of frames, on the relationship between frame bounds and density, and on the structure of the dual frame of a localized frame. These abstract results yield an array of new implications for irregular Gabor frames. Various Nyquist density results for Gabor frames are recovered as special cases, but in the process both their meaning and implications are clarified. New results are obtained on the excess and overcompleteness of Gabor frames, on the relationship between frame bounds and density, and on the structure of the dual frame of an irregular Gabor frame. More generally, these results apply both to Gabor frames and to systems of Gabor molecules, whose elements share only a common envelope of concentration in the time-frequency plane.
\end{abstract}

\section{INTRODUCTION}

Frames were first introduced by Duffin and Schaeffer DS52 in the context of nonharmonic Fourier series, and today frames play important roles in many applications in mathematics, science, and engineering, including time-frequency analysis Grö01, internet coding [GKK01, speech and music processing [WGW04, communication SH03, multiple antenna coding SHHS01, medicine UAL03, quantum computing [KR05, and many other areas.

Received by the editors July 10, 2005.

2000 Mathematics Subject Classification. Primary 42C15; Secondary 46C99.

Key words and phrases. Density, excess, frames, Gabor systems, modulation spaces, overcompleteness, Riesz bases, wavelets, Weyl-Heisenberg systems.

The second author was partially supported by NSF Grants DMS-0102686 and DMS-0405376.

The third author was partially supported by NSF Grant DMS-0139261.

The fourth author was partially supported by The City University of New York PSC-CUNY Research Award Program.

(C)2006 American Mathematical Society Reverts to public domain 28 years from publication 
The fundamental structural feature of a frame that is not a Riesz basis is the overcompleteness of its elements (i.e., after the deletion of a number of frame elements, the remaining set still spans the original space). To date, even partial understanding of this overcompleteness has been restricted to limited examples, such as finite-dimensional frames or regular frames of time-frequency shifts (lattice Gabor frames). The ideas and results presented here provide a quantitative framework for describing the overcompleteness of a large class of frames. The consequences of these ideas are: (a) an array of fundamental new results for frames that hold in a general setting, (b) significant new results for the case of irregular and lattice Gabor frames, as well as a new framing of existing results that clarifies their meaning, and (c) the presentation of a powerful new approach to density and overcompleteness which introduces a fruitful new area of research. Full details and proofs will appear in the papers BCHL06a, BCHL06b.

At the core of our main results is Theorem 3.3. The precise statement of the theorem requires some detailed notation, but the essence of the result can be summarized as follows. We begin with two frames $\mathcal{F}=\left\{f_{i}\right\}_{i \in I}$ and $\mathcal{E}=\left\{e_{j}\right\}_{j \in G}$, where $G$ is a discrete abelian group, and introduce a notion of the localization of $\mathcal{F}$ with respect to $\mathcal{E}$. The idea of localization is that it describes the decay of the coefficients of the expansion of elements of $\mathcal{F}$ in terms of the elements of $\mathcal{E}$. To make this notion of decay meaningful, a map $a$ from the index set $I$ into the index set $G$ is introduced. With this setup, Theorem 3.3 establishes a remarkable equality between three seemingly unrelated quantities: the relative measure of $\mathcal{F}$, the relative measure of $\mathcal{E}$ - both of which are determined by certain averages of inner products of frame elements with their corresponding dual frame elements - and the density of the set $a(I)$ in $G$. This equality between density and relative measure is striking, since the relative measure is a function of the frame elements, while the density is solely determined by the index set $I$ and the mapping $a: I \rightarrow G$.

We can summarize the impact of Theorem 3.3 and the other main results of this paper as follows.

Localization of frames. We provide a comprehensive analysis of the localization of frames. Localization is not a single concept, but a suite of related ideas. We introduce a collection of notions of localization as well as related approximation properties for frames. We provide a comprehensive examination of the interrelations between these localization and approximation concepts (Theorem 2.6).

Density and overcompleteness. We explore the implications of the connection between density and overcompleteness. We show that in any overcomplete frame which possesses sufficient localization, the overcompleteness must have a certain degree of uniformity. Specifically, we construct an infinite subset of the frame with positive density which can be removed yet still leave a frame (Theorem [3.6). We obtain relations among the frame bounds, density of the index set $I$, and norms of the frame elements, and prove in particular that if $\mathcal{F}$ is a tight localized frame whose elements all have the same norm, then the index set $I$ must have uniform density (Theorem 3.5)

Structure of the canonical dual frame. We explore the structure of the canonical dual frame, showing that if a frame is sufficiently localized, then its canonical dual frame is also localized (Theorem 2.8).

The Feichtinger conjecture for localized frames. We prove that any sufficiently localized frame can be written as a finite union of Riesz sequences (Theorem 3.7]). 
This shows that the Feichtinger conjecture (which has recently been shown to be equivalent to the famous Kadison-Singer conjecture [CT06]) is true for the case of localized frames.

Application to Gabor frames. We apply our theoretical results to the case of Gabor systems, yielding a collection of new results summarized as follows.

(a) Atoms with time-frequency concentration generate localized Gabor frames (Theorem 4.4). We show how the degree of localization of a Gabor frame is tied to the time-frequency concentration of the generating function or "atom". This alone yields a significant improvement over what was previously known about the approximation properties of irregular Gabor frames. We extend these results to more general systems of Gabor molecules whose elements are not required to be simple time-frequency shifts of each other, but instead need only share a common envelope of concentration about points in the time-frequency plane.

(b) Structure of the canonical dual frame (Theorem 4.9). While there have been several recent advances in the theory of irregular Gabor frames, e.g., Jan98, CFZ01, HW01, SZ02, LW03, until Grö04 essentially nothing has been known about the structure of the canonical dual of an irregular Gabor frame beyond the fact that it is some collection of elements of $L^{2}\left(\mathbf{R}^{d}\right)$. We prove that if an irregular Gabor frame is generated by a function $g$ which is sufficiently concentrated in the time-frequency plane (specifically, $g$ lies in the modulation space $M^{1}$ ), then the elements of the dual frame also lie in $M^{1}$. We further prove that the dual frame forms a set of Gabor molecules, and thus, while it need not form a Gabor frame, the elements do share a common envelope of concentration in the time-frequency plane. Moreover, this same result applies if the original frame was only itself a frame of Gabor molecules. This greatly extends a recent result of Gröchenig and Leinert GL04, which covered only the case of lattice Gabor frames.

(c) A relationship between density of time-frequency shifts and inner products of frame elements (Theorems 4.5, 4.6). As a consequence of Theorem 3.3. a remarkable equality between the density of the time-frequency shifts of a Gabor frame and certain averages of inner products between Gabor frame elements and the canonical dual frame elements is shown. This both recovers and extends known density results for Gabor frames.

(d) Excess of Gabor frames (Theorem 4.7). We show that a set of frame elements with positive density can be removed from any overcomplete Gabor frame.

Moreover, the results above extend with minor changes from irregular Gabor frames to frames of Gabor molecules.

We believe that localization is a powerful and useful new concept. We note that Gröchenig has independently introduced a concept of localized frames, for a completely different purpose Grö04. In his elegant paper, Gröchenig has shown that frames which are sufficiently localized in his sense provide frame expansions not only for the base Hilbert space $H$ but for an entire family of associated Banach spaces. He further showed that if a frame is sufficiently localized in his sense (a polynomial or exponential localization), then the dual frame is similarly localized. We learned of Gröchenig's results shortly after completion of our own major results. The definitions of localizations presented here and in Grö04 differ, but the fact 
that this single concept has independently arisen for two very distinct applications shows its utility.

1.1. General notation. $H$ will refer to a separable Hilbert space. The frame or system of interest will be indexed by a countable index set $I$. The reference frame or system will be indexed by an additive discrete group $G$ of the form

$$
G=\prod_{i=1}^{d} a_{i} \mathbf{Z} \times \prod_{j=1}^{e} \mathbf{Z}_{n_{j}}
$$

with a metric on $G$ defined as follows. Given $g=\left(a_{1} n_{1}, \ldots, a_{d} n_{d}, m_{1}, \ldots, m_{e}\right) \in G$, set

$$
|g|=\sup \left\{\left|a_{1} n_{1}\right|, \ldots,\left|a_{d} n_{d}\right|, \delta\left(m_{1}\right), \ldots, \delta\left(m_{e}\right)\right\}
$$

where $\delta\left(m_{j}\right)=0$ if $m_{j}=0$, otherwise $\delta\left(m_{j}\right)=1$. The metric is $d(g, h)=|g-h|$ for $g, h \in G$. The reader can simply take $G=\mathbf{Z}^{d}$ without much loss of insight on a first reading.

We implicitly assume that there exists a map $a: I \rightarrow G$ associated with $I$ and $G$. This map will often not be injective. For each integer $N>0$ we let

$$
S_{N}(j)=\left\{k \in G:|k-j| \leq \frac{N}{2}\right\}
$$

denote a discrete "cube" or "box" in $G$ centered at $j \in G$. We let $I_{N}(j)$ denote the inverse image of $S_{N}(j)$ under $a$, i.e.,

$$
I_{N}(j)=a^{-1}\left(S_{N}(j)\right)=\left\{i \in I: a(i) \in S_{N}(j)\right\}
$$

1.2. Notation for frames and Riesz bases. We use standard notation for frames and Riesz bases as found in the texts Chr03, Dau92, Grö01, You01.

A sequence $\mathcal{F}=\left\{f_{i}\right\}_{i \in I}$ is a frame for $H$ if there exist frame bounds $A, B>0$ such that $A\|f\|^{2} \leq \sum_{i \in I}\left|\left\langle f, f_{i}\right\rangle\right|^{2} \leq B\|f\|^{2}$ for all $f \in H$. The analysis operator $T: H \rightarrow \ell^{2}(I)$ is $T f=\left\{\left\langle f, f_{i}\right\rangle\right\}_{i \in I}$, and its adjoint $T^{*} c=\sum_{i \in I} c_{i} f_{i}$ is the synthesis operator. The Gram matrix is $T T^{*}=\left[\left\langle f_{i}, f_{j}\right\rangle\right]_{i, j \in I}$. The frame operator $S f=$ $T^{*} T f=\sum_{i \in I}\left\langle f, f_{i}\right\rangle f_{i}$ is a bounded, positive, and invertible mapping of $H$ onto itself. The canonical dual frame is $\tilde{\mathcal{F}}=S^{-1}(\mathcal{F})=\left\{\tilde{f}_{i}\right\}_{i \in I}$ where $\tilde{f}_{i}=S^{-1} f_{i}$. We call $\mathcal{F}$ a tight frame if we can take $A=B$, and a Parseval frame if we can take $A=B=1$. If $\mathcal{F}$ is any frame, then $S^{-1 / 2}(\mathcal{F})$ is the canonical Parseval frame associated to $\mathcal{F}$. We call $\mathcal{F}$ a uniform norm frame if all the frame elements have identical norms, i.e., if $\left\|f_{i}\right\|=$ const for all $i \in I$. A frame is norm-bounded below if $\inf _{i}\left\|f_{i}\right\|>0$.

A sequence which satisfies the upper frame bound estimate, but not necessarily the lower estimate, is called a Bessel sequence and $B$ is a Bessel bound.

A sequence $\mathcal{F}=\left\{f_{i}\right\}_{i \in I}$ that is a frame for its closed linear span in $H$ is called a frame sequence. In this case $\tilde{\mathcal{F}}=\left\{\tilde{f}_{i}\right\}_{i \in I}$ will denote its canonical dual frame within $\overline{\operatorname{span}}(F)$.

A frame is a basis if and only if it is a Riesz basis. A Riesz sequence is a sequence that forms a Riesz basis for its closed linear span in $H$. 


\section{Density, localization, HAP, And Relative measure}

2.1. Density. Given an index set $I$ and a map $a: I \rightarrow G$, we define the density of $I$ by computing the analogue of the Beurling density of its image $a(I)$ as a subset of $G$. Note that we regard $I$ as a sequence, and hence repetitions of images count in determining the density.

Definition 2.1 (Density). The lower and upper densities of I with respect to a are

$$
D^{-}(I, a)=\liminf _{N \rightarrow \infty} \inf _{j \in G} \frac{\left|I_{N}(j)\right|}{\left|S_{N}(j)\right|}, \quad D^{+}(I, a)=\limsup _{N \rightarrow \infty} \sup _{j \in G} \frac{\left|I_{N}(j)\right|}{\left|S_{N}(j)\right|},
$$

respectively. Note that $0 \leq D^{-}(I, a) \leq D^{+}(I, a) \leq \infty$. When $D^{-}(I, a)=$ $D^{+}(I, a)=D$, we say that $I$ has uniform density $D$.

These lower and upper densities are only the extremes of the possible densities that we could naturally assign to $I$ with respect to $a$. In particular, instead of taking the infimum or supremum over all possible centers in (2.1) we could choose one specific sequence of centers, and instead of computing the liminf or limsup we could consider the limit with respect to some ultrafilter. The different possible choices of ultrafilters and sequences of centers give the following natural collection of definitions of density.

Definition 2.2. Let $p$ be a free ultrafilter, and let $c=\left(c_{N}\right)_{N \in \mathbf{N}}$ be any sequence of centers $c_{N} \in G$. Then the density of $I$ with respect to $a, p$, and $c$ is

$$
D(p, c)=D(p, c ; I, a)=p \text {-lim } \frac{\left|I_{N}\left(c_{N}\right)\right|}{\left|S_{N}\left(c_{N}\right)\right|} .
$$

2.2. The localization properties. We introduce a collection of definitions of localization, given in terms of the decay of the inner products of the elements of one sequence $\mathcal{F}$ with respect to the elements of a reference sequence $\mathcal{E}$.

The words "column" and "row" in the following definition refer to the $I \times G$

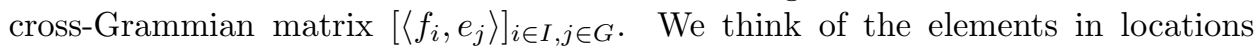
$(i, a(i))$ as corresponding to the main diagonal of this matrix.

Definition 2.3 (Localization). Let $\mathcal{F}=\left\{f_{i}\right\}_{i \in I}$ and $\mathcal{E}=\left\{e_{j}\right\}_{j \in G}$ be sequences in $H$.

(a) We say that $\mathcal{F}$ is $\ell^{p}$-localized with respect to the reference sequence $\mathcal{E}$ and the map $a$, or simply that $(\mathcal{F}, a, \mathcal{E})$ is $\ell^{p}$-localized, if

$$
\sum_{j \in G} \sup _{i \in I}\left|\left\langle f_{i}, e_{j+a(i)}\right\rangle\right|^{p}<\infty .
$$

Equivalently, there must exist an $r \in \ell^{p}(G)$ such that

$$
\forall i \in I, \quad \forall j \in G, \quad\left|\left\langle f_{i}, e_{j}\right\rangle\right| \leq r_{a(i)-j} .
$$

(b) $(\mathcal{F}, a, \mathcal{E})$ has $\ell^{p}$-column decay if for every $\varepsilon>0$ there is an integer $N_{\varepsilon}>0$ so that

$$
\forall j \in G, \quad \sum_{i \in I \backslash I_{N_{\varepsilon}}(j)}\left|\left\langle f_{i}, e_{j}\right\rangle\right|^{p}<\varepsilon .
$$

(c) $(\mathcal{F}, a, \mathcal{E})$ has $\ell^{p}$-row decay if for every $\varepsilon>0$ there is an integer $N_{\varepsilon}>0$ so that

$$
\forall i \in I, \quad \sum_{j \in G \backslash S_{N_{\varepsilon}}(a(i))}\left|\left\langle f_{i}, e_{j}\right\rangle\right|^{p}<\varepsilon .
$$


2.3. The approximation properties. The following approximation properties extract the essence of the homogeneous approximation property that is satisfied by Gabor frames (see [RS95, GR96, CDH99]), but without reference to the exact structure of Gabor frames.

Definition 2.4 (Homogeneous approximation properties). Let $\mathcal{F}=\left\{f_{i}\right\}_{i \in I}$ be a frame for $H$ with canonical dual $\tilde{\mathcal{F}}=\left\{\tilde{f}_{i}\right\}_{i \in I}$, and let $\mathcal{E}=\left\{e_{j}\right\}_{j \in G}$ be a sequence in $H$.

(a) $(\mathcal{F}, a, \mathcal{E})$ has the weak $H A P$ if for every $\varepsilon>0$, there is an integer $N_{\varepsilon}>0$ so that for every $j \in G$ we have $\operatorname{dist}\left(e_{j}, \overline{\operatorname{span}}\left\{\tilde{f}_{i}: i \in I_{N_{\varepsilon}}(j)\right\}\right)<\varepsilon$.

(b) $(\mathcal{F}, a, \mathcal{E})$ has the strong $H A P$ if for every $\varepsilon>0$, there is an integer $N_{\varepsilon}>0$ so that for every $j \in G$ we have

$$
\left\|e_{j}-\sum_{i \in I_{N_{\varepsilon}}(j)}\left\langle e_{j}, f_{i}\right\rangle \tilde{f}_{i}\right\|<\varepsilon
$$

Definition 2.5 (Dual homogeneous approximation properties). Let $\mathcal{F}=\left\{f_{i}\right\}_{i \in I}$ be a sequence in $H$, and let $\mathcal{E}=\left\{e_{j}\right\}_{j \in G}$ be a frame for $H$ with canonical dual $\tilde{\mathcal{E}}=\left\{\tilde{e}_{j}\right\}_{j \in G}$.

(a) $(\mathcal{F}, a, \mathcal{E})$ has the weak dual HAP if for every $\varepsilon>0$, there is an integer $N_{\varepsilon}>0$ so that for every $i \in I$ we have $\operatorname{dist}\left(f_{i}, \overline{\operatorname{span}}\left\{\tilde{e}_{j}: j \in S_{N_{\varepsilon}}(a(i))\right\}\right)<\varepsilon$.

(b) $(\mathcal{F}, a, \mathcal{E})$ has the strong dual HAP if for every $\varepsilon>0$, there is an integer $N_{\varepsilon}>0$ so that for every $i \in I$ we have $\left\|f_{i}-\sum_{j \in S_{N_{\varepsilon}}(a(i))}\left\langle f_{i}, e_{j}\right\rangle \tilde{e}_{j}\right\|<\varepsilon$.

2.4. Relations among the localization and approximation properties. The following theorem proved in BCHL06b summarizes the relationships that hold among the localization and approximation properties. We exhibit counterexamples in BCHL06b] to most of the converse implications.

Theorem 2.6. If $\mathcal{F}=\left\{f_{i}\right\}_{i \in I}$ and $\mathcal{E}=\left\{e_{j}\right\}_{j \in G}$ are sequences in $H$, then the following statements hold.

(a) If $\mathcal{F}$ is a frame for $H$, then $\ell^{2}$-column decay implies the strong HAP.

(b) If $\mathcal{F}$ is a frame for $H$ and $\sup _{j}\left\|e_{j}\right\|<\infty$, then the strong HAP implies $\ell^{2}$-column decay.

(c) If $\mathcal{E}$ is a frame for $H$, then $\ell^{2}$-row decay implies the strong dual HAP.

(d) If $\mathcal{E}$ is a frame for $H$ and $\sup _{i}\left\|f_{i}\right\|<\infty$, then the strong dual HAP implies $\ell^{2}$-row decay.

(e) If $\mathcal{F}$ is a frame for $H$, then the strong $H A P$ implies the weak HAP. If $\mathcal{F}$ is a Riesz basis for $H$, then the weak HAP implies the strong HAP.

(f) If $\mathcal{E}$ is a frame for $H$, then the strong dual HAP implies the weak dual HAP. If $\mathcal{E}$ is a Riesz basis for $H$, then the weak dual HAP implies the strong dual $H A P$.

(g) If $D^{+}(I, a)<\infty$ and $1 \leq p<\infty$, then $\ell^{p}$-localization implies both $\ell^{p}$ column and $\ell^{p}$-row decay.

For the case that $\mathcal{F}$ and $\mathcal{E}$ are both frames for $H$ and the upper density $D^{+}(I, a)$ is finite, these relations can be summarized in the diagram in Figure 1 .

2.5. Self-localization. It is also useful to consider localizations where the system $\mathcal{F}=\left\{f_{i}\right\}_{i \in I}$ is compared to itself or to its canonical dual frame instead of 


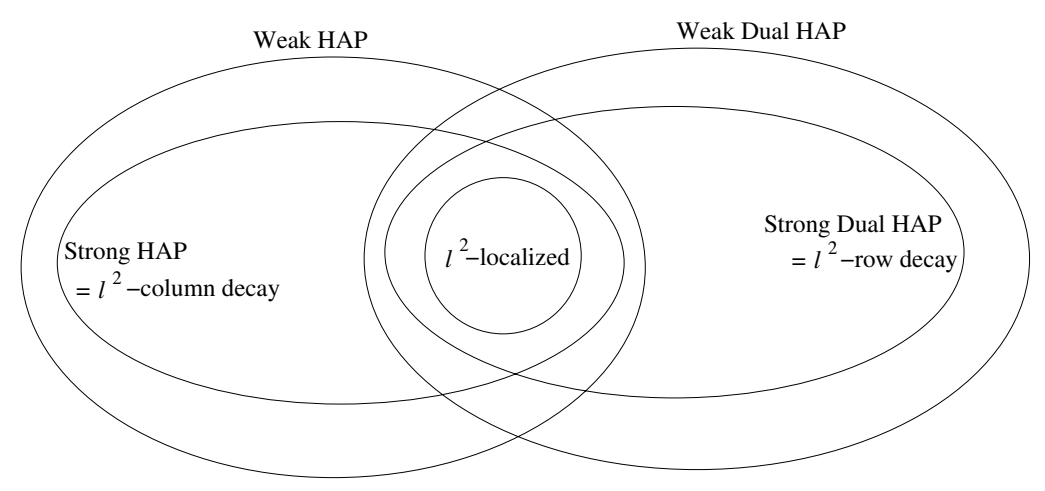

FiguRE 1. Relations among the localization and approximation properties for $p=2$, under the assumptions that $\mathcal{F}, \mathcal{E}$ are frames and $D^{+}(I, a)<\infty$.

to a reference system $\mathcal{E}$. An analogous polynomial or exponential "intrinsic localization" was independently introduced by Gröchenig in Grö03; see also For03, GF05. Although there is no reference system, we still require a mapping $a: I \rightarrow G$ associating $I$ with a group $G$.

Definition 2.7 (Self-localization). Let $\mathcal{F}=\left\{f_{i}\right\}_{i \in I}$ be a sequence in $H$.

(a) $(\mathcal{F}, a)$ is $\ell^{p}$-self-localized if there exists $r \in \ell^{p}(G)$ such that

$$
\forall i, j \in I, \quad\left|\left\langle f_{i}, f_{j}\right\rangle\right| \leq r_{a(i)-a(j)} .
$$

(b) If $\mathcal{F}$ is a frame sequence, then $(\mathcal{F}, a)$ is $\ell^{p}$-localized with respect to its canonical dual frame sequence $\tilde{\mathcal{F}}=\left\{\tilde{f}_{i}\right\}_{i \in I}$ if there exists $r \in \ell^{p}(G)$ such that

$$
\forall i, j \in I, \quad\left|\left\langle f_{i}, \tilde{f}_{j}\right\rangle\right| \leq r_{a(i)-a(j)} .
$$

We show in BCHL06b that $\ell^{1}$-localization with respect to the dual frame does not imply $\ell^{1}$-self-localization. However, the following result proved in BCHL06a states that the converse is true. The proof relies on an application of a type of noncommutative Wiener's lemma that was independently derived by Gohberg, Kaashoek, and Woerdeman [GKW89], Baskakov Bas90, and Sjöstrand [Sjö95.

Theorem 2.8. Let $\mathcal{F}=\left\{f_{i}\right\}_{i \in I}$ be a frame for $H$, and assume that $D^{+}(I, a)<\infty$. Let $\tilde{\mathcal{F}}$ be the canonical dual frame and $S^{-1 / 2}(\mathcal{F})$ the canonical Parseval frame. If $(\mathcal{F}, a)$ is $\ell^{1}$-self-localized, then:

(a) $(\mathcal{F}, a)$ is $\ell^{1}$-localized with respect to its canonical dual frame $\tilde{\mathcal{F}}=\left\{\tilde{f}_{i}\right\}_{i \in I}$,

(b) $(\tilde{\mathcal{F}}, a)$ is $\ell^{1}$-self-localized, and

(c) $\left(S^{-1 / 2}(\mathcal{F}), a\right)$ is $\ell^{1}$-self-localized.

2.6. Relative measure. We now define the relative measure of frame sequences.

Definition 2.9. (a) Let $\mathcal{F}=\left\{f_{i}\right\}_{i \in I}$ and $\mathcal{E}=\left\{e_{j}\right\}_{j \in G}$ be frame sequences in $H$. Let $P_{\mathcal{F}}, P_{\mathcal{E}}$ denote the orthogonal projections of $H$ onto $\overline{\operatorname{span}}(\mathcal{F})$ and $\overline{\operatorname{span}}(\mathcal{E})$, respectively. Then given a free ultrafilter $p$ and a sequence of centers $c=\left(c_{N}\right)_{N \in \mathbf{N}}$ in $G$, we define the relative measure of $\mathcal{F}$ with respect to $\mathcal{E}$, $p$, and $c$ to be

$$
\mathcal{M}_{\mathcal{E}}(\mathcal{F} ; p, c)=p \text {-lim } \frac{1}{\left|I_{N}\left(c_{N}\right)\right|} \sum_{i \in I_{N}\left(c_{N}\right)}\left\langle P_{\mathcal{E}} f_{i}, \tilde{f}_{i}\right\rangle .
$$


The relative measure of $\mathcal{E}$ with respect to $\mathcal{F}$ is

$$
\mathcal{M}_{\mathcal{F}}(\mathcal{E} ; p, c)=p \text {-lim } \frac{1}{N \in \mathbf{N}} \sum_{\left|S_{N}\left(c_{N}\right)\right|}\left\langle P_{\mathcal{F}} \tilde{e}_{j}, e_{j}\right\rangle .
$$

(b) If $\overline{\operatorname{span}}(\mathcal{E}) \supset \overline{\operatorname{span}}(\mathcal{F})$, then $P_{\mathcal{E}}$ is the identity map and $\mathcal{E}$ plays no role in determining the value of $\mathcal{M}_{\mathcal{E}}(\mathcal{F} ; p, e)$. Therefore, in this case we define the measure of $\mathcal{F}$ with respect to $p$ and $c$ to be

$$
\mathcal{M}(\mathcal{F} ; p, c)=p-\lim _{N \in \mathbf{N}} \frac{1}{\left|I_{N}\left(c_{N}\right)\right|} \sum_{i \in I_{N}\left(c_{N}\right)}\left\langle f_{i}, \tilde{f}_{i}\right\rangle .
$$

Since $0 \leq\left\langle f_{i}, \tilde{f}_{i}\right\rangle \leq 1$ for all $i$, it follows that $0 \leq \mathcal{M}(\mathcal{F} ; p, c) \leq 1$.

We further define the lower and upper measures of $\mathcal{F}$ by

$$
\begin{aligned}
& \mathcal{M}^{-}(\mathcal{F})=\liminf _{N \rightarrow \infty} \inf _{j \in G} \frac{1}{\left|I_{N}(j)\right|} \sum_{i \in I_{N}(j)}\left\langle f_{i}, \tilde{f}_{i}\right\rangle, \\
& \mathcal{M}^{+}(\mathcal{F})=\limsup _{N \rightarrow \infty} \sup _{j \in G} \frac{1}{\left|I_{N}(j)\right|} \sum_{i \in I_{N}(j)}\left\langle f_{i}, \tilde{f}_{i}\right\rangle .
\end{aligned}
$$

(c) When $\overline{\operatorname{span}}(\mathcal{F}) \supset \overline{\operatorname{span}}(\mathcal{E})$, we define the measures $\mathcal{M}(\mathcal{E} ; p, c)$ and $\mathcal{M}^{ \pm}(\mathcal{E})$ in an analogous manner.

Example 2.10. If $\overline{\operatorname{span}}(\mathcal{E}) \supset \overline{\operatorname{span}}(\mathcal{F})$ and $\mathcal{F}$ is a Riesz sequence, then $\left\langle f_{i}, \tilde{f}_{i}\right\rangle=1$ for every $i \in I$, so $\mathcal{M}(\mathcal{F} ; p, c)=\mathcal{M}^{+}(\mathcal{F})=\mathcal{M}^{-}(\mathcal{F})=1$.

Likewise, if $\overline{\operatorname{span}}(\mathcal{F}) \supset \overline{\operatorname{span}}(\mathcal{E})$ and $\mathcal{E}$ is a Riesz sequence, then $\left\langle\tilde{e}_{j}, e_{j}\right\rangle=1$ for every $j \in G$, so $\mathcal{M}(\mathcal{E} ; p, c)=\mathcal{M}^{+}(\mathcal{E})=\mathcal{M}^{-}(\mathcal{E})=1$.

\section{Density And overcompleteness}

3.1. Necessary density conditions. Following are two necessary conditions on the density of localized frames proved in BCHL06a.

Theorem 3.1 (Necessary density bounds).

(a) Assume $\mathcal{F}=\left\{f_{i}\right\}_{i \in I}$ is a frame for $H$ and $\mathcal{E}=\left\{e_{j}\right\}_{j \in G}$ is a Riesz sequence in $H$. If $(\mathcal{F}, a, \mathcal{E})$ has the weak $H A P$, then

$$
1 \leq D^{-}(I, a) \leq D^{+}(I, a) \leq \infty .
$$

(b) Assume $\mathcal{F}=\left\{f_{i}\right\}_{i \in I}$ is a Riesz sequence in $H$ and $\mathcal{E}=\left\{e_{j}\right\}_{j \in G}$ is a frame for $H$. If $(\mathcal{F}, a, \mathcal{E})$ has the weak dual HAP, then

$$
0 \leq D^{-}(I, a) \leq D^{+}(I, a) \leq 1 .
$$

Theorem 3.2 (Necessary finite density condition). Let $\mathcal{F}=\left\{f_{i}\right\}_{i \in I}$ be a Bessel sequence in $H$, and suppose $\inf _{i \in I}\left\|f_{i}\right\|>0$. Assume $\mathcal{E}=\left\{e_{j}\right\}_{j \in G}$ is a frame for $H$. If $(\mathcal{F}, a, \mathcal{E})$ has $\ell^{2}$-row decay, then $D^{+}(I, a)<\infty$.

3.2. The connection between density and relative measure. The following theorem proved in BCHL06a] presents the fundamental relationship between density and relative measure for localized frames.

Theorem 3.3 (Density-relative measure). Let $\mathcal{F}=\left\{f_{i}\right\}_{i \in I}$ and $\mathcal{E}=\left\{e_{j}\right\}_{j \in G}$ be frame sequences in $H$. If $D^{+}(I, a)<\infty$ and $(\mathcal{F}, a, \mathcal{E})$ has both $\ell^{2}$-column decay and 
$\ell^{2}$-row decay, then for every sequence of centers $c=\left(c_{N}\right)_{N \in \mathbf{N}}$ in $G$ and any free ultrafilter $p$,

$$
\mathcal{M}_{\mathcal{F}}(\mathcal{E} ; p, c)=D(p, c) \cdot \mathcal{M}_{\mathcal{E}}(\mathcal{F} ; p, c) .
$$

Specializing to the case where $\mathcal{F}$ and $\mathcal{E}$ are both frames for $H$ yields the following result [BCHL06a].

Theorem 3.4 (Abstract density theorem). Let $\mathcal{F}=\left\{f_{i}\right\}_{i \in I}$ and $\mathcal{E}=\left\{e_{j}\right\}_{j \in G}$ be frames for $H$, and suppose that $D^{+}(I, a)<\infty$. If $(\mathcal{F}, a, \mathcal{E})$ has both $\ell^{2}$-column decay and $\ell^{2}$-row decay, then the following statements hold.

(a) For each free ultrafilter $p$ and sequence of centers $c=\left(c_{N}\right)_{N \in \mathbf{N}}$ in $G$, we have $\mathcal{M}(\mathcal{E} ; p, c)=D(p, c) \cdot \mathcal{M}(\mathcal{F} ; p, c)$. Consequently,

$$
\begin{aligned}
& \mathcal{M}^{-}(\mathcal{E}) \leq D^{+}(I, a) \cdot \mathcal{M}^{-}(\mathcal{F}) \leq \mathcal{M}^{+}(\mathcal{E}), \\
& \mathcal{M}^{-}(\mathcal{E}) \leq D^{-}(I, a) \cdot \mathcal{M}^{+}(\mathcal{F}) \leq \mathcal{M}^{+}(\mathcal{E}) .
\end{aligned}
$$

(b) If $D^{+}(I, a)>\mathcal{M}^{+}(\mathcal{E})$, then there exists an infinite set $J \subset I$ such that $\left\{f_{i}\right\}_{i \in I \backslash J}$ is still a frame for $H$.

If $\mathcal{E}$ is a Riesz basis for $H$, then the following additional statements hold.

(c) For each free ultrafilter $p$ and sequence of centers $c=\left(c_{N}\right)_{N \in \mathbf{N}}$ in $G$, we have

$$
\mathcal{M}(\mathcal{F} ; p, c)=\frac{1}{D(p, c)}, \quad \mathcal{M}^{-}(\mathcal{F})=\frac{1}{D^{+}(I, a)}, \quad \mathcal{M}^{+}(\mathcal{F})=\frac{1}{D^{-}(I, a)}
$$

(d) $D^{-}(I, a) \geq 1$

(e) If $D^{+}(I, a)>1$, then there exists an infinite subset $J \subset I$ such that $\left\{f_{i}\right\}_{i \in I \backslash J}$ is still a frame for $H$.

(f) If $\mathcal{F}$ is also a Riesz basis for $H$, then for each free ultrafilter $p$ and sequence of centers $c=\left(c_{N}\right)_{N \in \mathbf{N}}$ in $G$, we have

$$
\begin{aligned}
& D^{-}(I, a)=D(p, c)=D^{+}(I, a)=1, \\
& \mathcal{M}^{-}(\mathcal{F})=\mathcal{M}(\mathcal{F} ; p, c)=\mathcal{M}^{+}(\mathcal{F})=1 .
\end{aligned}
$$

Next we derive new relationships among the density, frame bounds, and norms of the frame elements for localized frames [BCHL06a. In particular, if $\mathcal{F}$ and $\mathcal{E}$ are both tight uniform norm frames, then the index set $I$ must have uniform density.

Theorem 3.5 (Density-frame bounds). Let $\mathcal{F}=\left\{f_{i}\right\}_{i \in I}$ be a frame for $H$ with frame bounds $A, B$, and let $\mathcal{E}=\left\{e_{j}\right\}_{j \in G}$ be a frame for $H$ with frame bounds $E, F$. If $D^{+}(I, a)<\infty$ and $(\mathcal{F}, a, \mathcal{E})$ has both $\ell^{2}$-column decay and $\ell^{2}$-row decay, then the following statements hold.

(a) For each free ultrafilter $p$ and sequence of centers $c=\left(c_{N}\right)_{N \in \mathbf{N}}$ in $G$,

$$
\begin{aligned}
& \frac{1}{F} p_{N \in \mathbf{N}} \frac{1}{\left|S_{N}\left(c_{N}\right)\right|} \sum_{j \in S_{N}\left(c_{N}\right)}\left\|e_{j}\right\|^{2} \leq \frac{D(p, c)}{A} p \text {-lim } \frac{1}{\left|I_{N}\left(c_{N}\right)\right|} \sum_{i \in I_{N}\left(c_{N}\right)}\left\|f_{i}\right\|^{2} \text {, } \\
& \frac{1}{E} p_{N \in \mathbf{N}} \frac{1}{\left|S_{N}\left(c_{N}\right)\right|} \sum_{j \in S_{N}\left(c_{N}\right)}\left\|e_{j}\right\|^{2} \geq \frac{D(p, c)}{B} p_{N \in \mathbf{N}} \lim \frac{1}{\left|I_{N}\left(c_{N}\right)\right|} \sum_{i \in I_{N}\left(c_{N}\right)}\left\|f_{i}\right\|^{2} .
\end{aligned}
$$

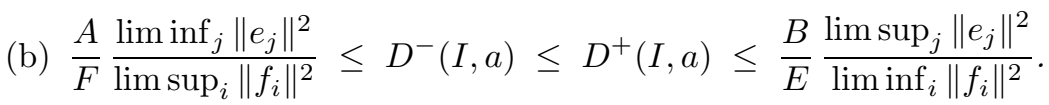


(c) If $\mathcal{F}$ and $\mathcal{E}$ are both uniform norm frames, with $\left\|f_{i}\right\|^{2}=\mathcal{N}_{\mathcal{F}}$ for $i \in I$ and $\left\|e_{j}\right\|^{2}=\mathcal{N}_{\mathcal{E}}$ for $j \in G$, then

$$
\frac{A \mathcal{N}_{\mathcal{E}}}{F \mathcal{N}_{\mathcal{F}}} \leq D^{-}(I, a) \leq D^{+}(I, a) \leq \frac{B \mathcal{N}_{\mathcal{E}}}{E \mathcal{N}_{\mathcal{F}}}
$$

Consequently, if $\mathcal{F}$ and $\mathcal{E}$ are both tight uniform norm frames, then $I$ has uniform density, with $D^{-}(I, a)=D^{+}(I, a)=\left(A \mathcal{N}_{\mathcal{E}}\right) /\left(E \mathcal{N}_{\mathcal{F}}\right)$.

3.3. Removing sets of positive measure. The following result states that in any sufficiently localized overcomplete frame, there is a subset of positive measure may be removed yet still leave a frame BCHL06a].

Theorem 3.6 (Positive uniform density removal). Let $\mathcal{F}=\left\{f_{i}\right\}_{i \in I}$ be a frame sequence with frame bounds $A, B$, and assume that the following statements hold:

(a) $0<D^{-}(I, a) \leq D^{+}(I, a)<\infty$,

(b) $(\mathcal{F}, a)$ is $\ell^{1}$-localized with respect to its canonical dual frame, and

(c) $\mathcal{M}^{+}(\mathcal{F})<1$.

Then there exists a subset $J \subset I$ such that $D^{+}(J, a)=D^{-}(J, a)>0$ and $\mathcal{F}_{I \backslash J}=$ $\left\{f_{i}\right\}_{i \in I \backslash J}$ is a frame for $\overline{\operatorname{span}}(\mathcal{F})$.

3.4. Localized frames and $\varepsilon$-Riesz sequences. Feichtinger has conjectured that every frame that is norm-bounded below can be written as a union of a finite number of Riesz sequences (systems that are Riesz bases for their closed linear spans). It is shown in [CCLV05], CT06] that Feichtinger's conjecture is equivalent to the celebrated Kadison-Singer (paving) conjecture, and that both of these are equivalent to a conjectured generalization of the Bourgain-Tzafriri restricted invertibility theorem.

The following result BCHL06a states that every norm-bounded frame that is $\ell^{1}$-self-localized is a finite union of $\varepsilon$-Riesz sequences. A Riesz sequence $\left\{f_{i}\right\}_{i \in I}$ is an $\varepsilon$-Riesz sequence if there exists a constant $A>0$ such that for every sequence $\left(c_{i}\right)_{i \in I} \in \ell^{2}(I)$ we have

$$
(1-\varepsilon) A \sum_{i \in I}\left|c_{i}\right|^{2} \leq\left\|\sum_{i \in I} c_{i} f_{i}\right\|^{2} \leq(1+\varepsilon) A \sum_{i \in I}\left|c_{i}\right|^{2} .
$$

Theorem 3.7. Let $\mathcal{F}=\left\{f_{i}\right\}_{i \in I}$ be a sequence in $H$. If

(a) $\left(\mathcal{F}\right.$, a) is $\ell^{1}$-self-localized,

(b) $D^{+}(I, a)<\infty$, and

(c) $\inf _{i}\left\|f_{i}\right\|>0$,

then for each $0<\varepsilon<\inf _{i}\left\|f_{i}\right\|, \mathcal{F}$ can be written as a finite union of $\varepsilon$-Riesz sequences.

\section{Applications to Gabor systems}

In this section we present new results on the properties of arbitrary or "irregular" Gabor systems.

For simplicity of presentation, most of our results will be stated for the case of Gabor frames for all of $L^{2}\left(\mathbf{R}^{d}\right)$, but most can be extended to the case of Gabor frame sequences, or to Gabor frames with multiple generators, by making use of the machinery developed in BCHL06a, BCHL06b. Moreover, as shown in BCHL06b, most of the results stated here can be extended from Gabor frames to more general frames of Gabor molecules (see Definition 4.8). 
4.1. Gabor systems and the reference system. A generic Gabor system generated by a function $g \in L^{2}\left(\mathbf{R}^{d}\right)$ and a sequence $\Lambda \subset \mathbf{R}^{2 d}$ will be written in any of the following forms:

$$
\mathcal{G}(g, \Lambda)=\left\{M_{\omega} T_{x} g\right\}_{(x, \omega) \in \Lambda}=\left\{e^{2 \pi i \omega \cdot t} g(t-x)\right\}_{(x, \omega) \in \Lambda}=\left\{g_{\lambda}\right\}_{\lambda \in \Lambda} .
$$

In the case that $\mathcal{G}(g, \Lambda)$ is a frame sequence we let

$$
\tilde{\mathcal{G}}=\left\{\tilde{g}_{\lambda}\right\}_{\lambda \in \Lambda}
$$

denote the canonical dual frame sequence, but it is important to note that while $g_{\lambda}$ is a time-frequency shift of $g$, it need not be the case that the functions $\tilde{g}_{\lambda}$ are time-frequency shifts of a single function. We address the question of the structure of the dual frame in more detail in Section 4.6

Our reference systems will be lattice Gabor systems indexed by the group

$$
G=\alpha \mathbf{Z}^{d} \times \beta \mathbf{Z}^{d},
$$

where $\alpha, \beta>0$ are fixed scalars. That is, our reference systems have the form

$$
\mathcal{G}(\phi, G)=\mathcal{G}\left(\phi, \alpha \mathbf{Z}^{d} \times \beta \mathbf{Z}^{d}\right)=\left\{M_{\eta} T_{u} \phi\right\}_{(\eta, u) \in G}=\left\{M_{\beta n} T_{\alpha k} \phi\right\}_{k, n \in \mathbf{Z}^{d}} .
$$

The canonical dual frame of a lattice Gabor frame sequence is another lattice Gabor frame sequence $\mathcal{G}(\tilde{\phi}, G)$, generated by some dual window $\tilde{\phi} \in L^{2}\left(\mathbf{R}^{d}\right)$. Usually the reference system makes an appearance only during the course of a proof, and does not appear in the statement of most of the theorems.

4.2. Cubes and the $a$ mapping. A natural map $a: \Lambda \rightarrow G$ is rounding to a near element of $G$, i.e.,

$$
a(x, \omega)=\left(\alpha \operatorname{Int}\left(\frac{x}{\alpha}\right), \beta \operatorname{Int}\left(\frac{\omega}{\beta}\right)\right), \quad(x, \omega) \in \Lambda,
$$

where $\operatorname{Int}(x)=\left(\left\lfloor x_{1}\right\rfloor, \ldots,\left\lfloor x_{d}\right\rfloor\right)$.

Given $z=(x, y) \in \mathbf{R}^{2 d}$, let $Q_{r}(z)=Q_{r}(x, y)$ denote the closed cube in $\mathbf{R}^{2 d}$ centered at $z$ with side length $r$. Then given $j \in G=\alpha \mathbf{Z}^{d} \times \beta \mathbf{Z}^{d}$, we have

$$
S_{N}(j)=G \cap Q_{N}(j) \quad \text { and } \quad I_{N}(j)=a^{-1}\left(G \cap Q_{N}(j)\right) .
$$

Note that $I_{N}(j)$ is very nearly $\Lambda \cap Q_{N}(j)$, except for the effect of rounding off points via the $a$ map. Thus

$$
\begin{aligned}
\left|S_{N}(j)\right| & =\left|G \cap Q_{N}(j)\right| \approx(\alpha \beta)^{-d} N^{2 d}, \\
\left|I_{N}(j)\right| & =\left|a^{-1}\left(G \cap Q_{N}(j)\right)\right| \approx\left|\Lambda \cap Q_{N}(j)\right| .
\end{aligned}
$$

4.3. Density and measure. Let $D_{B}^{+}(\Lambda)$ denote the standard upper Beurling density of $\Lambda$. Then this is related to our definition of the upper density of $\Lambda$ with respect to $a$ as follows:

$$
\begin{aligned}
D_{B}^{+}(\Lambda) & =\limsup _{N \rightarrow \infty} \sup _{j \in \mathbf{R}^{2 d}} \frac{\left|\Lambda \cap Q_{N}(j)\right|}{N^{2 d}} \\
& =\frac{1}{(\alpha \beta)^{d}} \limsup _{N \rightarrow \infty} \sup _{j \in \alpha \mathbf{Z}^{d} \times \beta \mathbf{Z}^{d}} \frac{\left|I_{N}(j)\right|}{\left|S_{N}(j)\right|}=\frac{1}{(\alpha \beta)^{d}} D^{+}(\Lambda, a) .
\end{aligned}
$$


Similarly the lower Beurling density of $\Lambda$ is $D_{B}^{-}(\Lambda)=(\alpha \beta)^{-d} D^{-}(\Lambda, a)$. In light of this, we define the Beurling density of $\Lambda$ with respect to a free ultrafilter $p$ and a sequence of centers $c=\left(c_{N}\right)_{N \in \mathbf{N}}$ in $\mathbf{R}^{2 d}$ to be

$$
D_{B}(\Lambda ; p, c)=(\alpha \beta)^{-d} D(\Lambda, a ; p, c)=(\alpha \beta)^{-d} p-\lim _{N \in \mathbf{N}} \frac{\left|a^{-1}\left(G \cap Q_{N}\left(c_{N}\right)\right)\right|}{\left|G \cap Q_{N}\left(c_{N}\right)\right|} .
$$

Our results for Gabor systems will all be stated in terms of these Beurling densities.

The measure of a Gabor frame sequence $\mathcal{G}(g, \Lambda)$ with respect to a free ultrafilter $p$ and a sequence of centers $c=\left(c_{N}\right)_{N \in \mathbf{N}}$ in $\mathbf{R}^{2 d}$ is

$$
\mathcal{M}(\mathcal{G}(g, \Lambda) ; p, c)=p-\lim _{N \in \mathbf{N}} \frac{1}{\left|a^{-1}\left(G \cap Q_{N}\left(c_{N}\right)\right)\right|} \sum_{a^{-1}\left(G \cap Q_{N}\left(c_{N}\right)\right)}\left\langle g_{\lambda}, \tilde{g}_{\lambda}\right\rangle .
$$

By making the approximations in (4.1) precise, we can reformulate the above quantities so that it is clear that the density and measure do not depend on the choice of $\alpha, \beta$ (analogous reformulations of the upper and lower density and measures also hold under the same hypotheses).

Lemma 4.1. Let $\Lambda \subset \mathbf{R}^{2 d}$ be given.

(a) If $D_{B}^{+}(\Lambda)<\infty$, then for any ultrafilter $p$ and any sequence of centers $c=$ $\left(c_{N}\right)_{N \in \mathbf{N}}$ in $\mathbf{R}^{2 d}$,

$$
D_{B}(\Lambda ; p, c)=p-\lim _{N \in \mathbf{N}} \frac{\left|\Lambda \cap Q_{N}\left(c_{N}\right)\right|}{N^{2 d}}
$$

(b) Let $g \in L^{2}\left(\mathbf{R}^{d}\right)$ be given. If $0<D_{B}^{-}(\Lambda) \leq D_{B}^{+}(\Lambda)<\infty$, then for any ultrafilter $p$ and any sequence of centers $c=\left(c_{N}\right)_{N \in \mathbf{N}}$ in $\mathbf{R}^{2 d}$,

$$
\mathcal{M}(\mathcal{G}(g, \Lambda) ; p, c)=p-\lim _{N \in \mathbf{N}} \frac{1}{\left|\Lambda \cap Q_{N}\left(c_{N}\right)\right|} \sum_{\lambda \in \Lambda \cap Q_{N}\left(c_{N}\right)}\left\langle g_{\lambda}, \tilde{g}_{\lambda}\right\rangle .
$$

4.4. Localization of Gabor systems. For most applications in time-frequency analysis, the generator of a Gabor system must possess some amount of joint concentration in both time and frequency. Concentration is quantified by the norms of the modulation spaces, which are the Banach function spaces naturally associated to time-frequency analysis. The modulation spaces were introduced and extensively studied by Feichtinger, e.g., Fei81, FG89a, FG89b. We refer to Grö01 for detailed discussion of the modulation spaces and references to the original literature. For our purposes, the following special case of unweighted modulation spaces will be sufficient.

\section{Definition 4.2.}

(a) The Short-Time Fourier Transform (STFT) of a tempered distribution $g \in \mathcal{S}^{\prime}\left(\mathbf{R}^{d}\right)$ with respect to a window function $\phi \in \mathcal{S}\left(\mathbf{R}^{d}\right)$ is

$$
V_{\phi} g(x, \omega)=\left\langle g, M_{\omega} T_{x} \phi\right\rangle, \quad(x, \omega) \in \mathbf{R}^{2 d} .
$$

(b) Let $\gamma(x)=2^{d / 4} e^{-\pi x \cdot x}$ be the Gaussian function. Then for $1 \leq p \leq \infty$, the modulation space $M^{p}\left(\mathbf{R}^{d}\right)$ consists of all tempered distributions $f \in \mathcal{S}^{\prime}\left(\mathbf{R}^{d}\right)$ such that

$$
\|f\|_{M^{p}}=\left\|V_{\gamma} f\right\|_{L^{p}}=\left(\iint_{\mathbf{R}^{2 d}}\left|\left\langle f, M_{\omega} T_{x} \gamma\right\rangle\right|^{p} d x d \omega\right)^{1 / p}<\infty .
$$


$M^{p}$ is a Banach space for each $1 \leq p \leq \infty$, and any nonzero function $g \in M^{1}$ can be substituted for $\gamma$ in (4.2) to define an equivalent norm for $M^{p}$. We have $M^{2}=L^{2}$, and $\mathcal{S} \subsetneq M^{p} \subsetneq M^{q} \subsetneq \mathcal{S}^{\prime}$ for $1 \leq p<q \leq \infty$, where $\mathcal{S}$ is the Schwartz class. The box function $\chi_{[0,1]}$ lies in $M^{p}$ for $p>1$, but is not in $M^{1}$.

In addition to the modulation spaces, we will also need the following special case of the Wiener amalgam spaces on $\mathbf{R}^{2 d}$. A comprehensive theory of amalgam spaces on locally compact groups was introduced by Feichtinger, starting with [Fei80]. We refer to Hei03 for an introduction to Wiener amalgams on Euclidean space, with extensive references to the original literature.

Definition 4.3. Given $1 \leq p \leq \infty$, the Wiener amalgam $W\left(\mathcal{C}, \ell^{p}\right)$ consists of all continuous functions $F$ on $\mathbf{R}^{2 d}$ for which

$$
\|F\|_{W\left(\mathcal{C}, \ell^{p}\right)}=\left(\sum_{(k, n) \in \mathbf{Z}^{2 d}} \sup _{(u, \eta) \in \mathbf{Q}_{\alpha, \beta}(\alpha k, \beta n)}|F(u, \eta)|^{p}\right)^{1 / p}<\infty,
$$

where $\mathbf{Q}_{\alpha, \beta}(x, y)=[0, \alpha)^{d} \times[0, \beta)^{d}+(x, y)$.

$W\left(\mathcal{C}, \ell^{p}\right)$ is a Banach space, and its definition is independent of the values of $\alpha$ and $\beta$ in the sense that each choice of $\alpha, \beta$ yields an equivalent norm for $W\left(\mathcal{C}, \ell^{p}\right)$.

The next result shows that if the generator of our reference system is an $M^{1}$ function $\phi$, then $(\mathcal{G}(g, \Lambda), a, \mathcal{G}(\phi, G))$ is $\ell^{p}$-localized whenever $g \in M^{p}$ [BCHL06b].

Theorem 4.4. Let $g \in L^{2}\left(\mathbf{R}^{d}\right)$ and $\Lambda \subset \mathbf{R}^{2 d}$ be given. Let $\phi \in L^{2}\left(\mathbf{R}^{d}\right)$ and $\alpha$, $\beta>0$ be given, and fix $1 \leq p \leq 2$. Then the following statements hold.

(a) If $g \in M^{p}$ and $\phi \in M^{1}$, then $(\mathcal{G}(g, \Lambda), a, \mathcal{G}(\phi, G))$ is $\ell^{p}$-localized.

(b) Suppose $\phi \in M^{1}$ and $\alpha, \beta>0$ are such that $\mathcal{G}(\phi, G)$ is a frame for $L^{2}\left(\mathbf{R}^{d}\right)$. If $(\mathcal{G}(g, \Lambda), a, \mathcal{G}(\phi, G))$ is $\ell^{p}$-localized, then $g \in M^{p}$.

(c) If $g \in M^{1}$ and $\phi \in M^{p}$, then $(\mathcal{G}(g, \Lambda), a, \mathcal{G}(\phi, G))$ is $\ell^{p}$-localized.

(d) If $g \in M^{1}$, then $(\mathcal{G}(g, \Lambda), a)$ is $\ell^{1}$-self-localized.

4.5. Density and overcompleteness for Gabor systems. Parts (b) and (c) of the following theorem are new results for Gabor frames and give a new interpretation of the density in terms of the measure of the frame BCHL06b]. Parts (a) and (d) recover the known density facts for irregular Gabor frames. Part (e) is the special case of lattice systems, and is related to the Wexler-Raz conditions for lattice Gabor frames [Jan95], DLL95].

Theorem 4.5. Let $g \in L^{2}\left(\mathbf{R}^{d}\right)$ and $\Lambda \subset \mathbf{R}^{2 d}$ be such that $\mathcal{G}(g, \Lambda)$ is a Gabor frame for $L^{2}\left(\mathbf{R}^{d}\right)$. Then the following statements hold.

(a) $1 \leq D_{B}^{-}(\Lambda) \leq D_{B}^{+}(\Lambda)<\infty$.

(b) For any free ultrafilter $p$ and any sequence of centers $c=\left(c_{N}\right)_{N \in \mathbf{N}}$ in $\mathbf{R}^{d}$,

$$
\mathcal{M}(\mathcal{G}(g, \Lambda) ; p, c)=\frac{1}{D_{B}(\Lambda ; p, c)} .
$$

(c) $\mathcal{M}^{-}(\mathcal{G}(g, \Lambda))=\frac{1}{D_{B}^{+}(\Lambda)}$ and $\mathcal{M}^{+}(\mathcal{G}(g, \Lambda))=\frac{1}{D_{B}^{-}(\Lambda)}$.

(d) If $\mathcal{G}(g, \Lambda)$ is a Riesz basis, then $D_{B}^{-}(\Lambda)=D_{B}^{+}(\Lambda)=1$.

(e) If $\Lambda=\alpha \mathbf{Z}^{d} \times \beta \mathbf{Z}^{d}$ then $0<\alpha \beta \leq 1$ and $\langle g, \tilde{g}\rangle=(\alpha \beta)^{d}$.

The following result derives new relationships between the density, frame bounds, and norm of the generator of an arbitrary Gabor frame BCHL06b. The special case of lattice systems was proved by Daubechies [Dau90, Eq. 2.2.9]. 
Theorem 4.6. Let $g \in L^{2}\left(\mathbf{R}^{d}\right)$ and $\Lambda \subset \mathbf{R}^{2 d}$ be such that $\mathcal{G}(g, \Lambda)$ is a Gabor frame for $L^{2}\left(\mathbf{R}^{d}\right)$, with frame bounds $A, B$. Then the following statements hold.

(a) $A \leq D_{B}^{-}(\Lambda)\|g\|_{2}^{2} \leq D_{B}^{+}(\Lambda)\|g\|_{2}^{2} \leq B$.

(b) If $\mathcal{G}(g, \Lambda)$ is a tight frame, then $\Lambda$ has uniform Beurling density, that is, $D_{B}^{-}(\Lambda)=D_{B}^{+}(\Lambda)$, and furthermore $A=D_{B}^{ \pm}(\Lambda)\|g\|_{2}^{2}$.

(c) If $\Lambda=\alpha \mathbf{Z}^{d} \times \beta \mathbf{Z}^{d}$, then $A \leq \frac{\|g\|_{2}^{2}}{(\alpha \beta)^{d}} \leq B$.

The following result states that subsets with positive density may be removed from an overcomplete Gabor frame yet still leave a frame BCHL06b.

Theorem 4.7. Let $g \in L^{2}\left(\mathbf{R}^{d}\right)$ and $\Lambda \subset \mathbf{R}^{2 d}$ be such that $\mathcal{G}(g, \Lambda)$ is a Gabor frame for $L^{2}\left(\mathbf{R}^{d}\right)$. If $g \in M^{1}$ and $D_{B}^{-}(\Lambda)>1$, then there exists $J \subset \Lambda$ with $D_{B}^{+}(J)=D_{B}^{-}(J)>0$ such that $\mathcal{G}(g, \Lambda \backslash J)$ is a frame for $L^{2}\left(\mathbf{R}^{d}\right)$.

4.6. Localization and structure of the canonical dual frame. The term "molecule" in the following definition arises from the convention that the generator $g$ of a Gabor system $\mathcal{G}(g, \Lambda)$ is often referred to as an "atom."

Definition 4.8. Let $\Lambda \subset \mathbf{R}^{2 d}$ and $f_{\lambda} \in L^{2}\left(\mathbf{R}^{d}\right)$ for $\lambda \in \Lambda$ be given. Then $\mathcal{F}=$ $\left\{f_{\lambda}\right\}_{\lambda \in \Lambda}$ is a set of Gabor molecules if there exists an envelope function $\Gamma \in W\left(\mathcal{C}, \ell^{2}\right)$ such that for every $\lambda \in \Lambda$ and $z \in \mathbf{R}^{2 d}$ we have $\left|V_{\gamma} f_{\lambda}(z)\right| \leq \Gamma(z-\lambda)$.

Thus, if $\Gamma$ is concentrated around the origin in $\mathbf{R}^{2 d}$, then the STFT of $f_{\lambda}$ is concentrated around the point $\lambda$. Every Gabor system $\mathcal{G}(g, \Lambda)$ is a set of Gabor molecules, as $\left|V_{\gamma} g_{\lambda}(z)\right|=\left|V_{\gamma} g(z-\lambda)\right|$ for every $z, \lambda$. It can be shown that the definition of Gabor molecules is unchanged if we replace the Gaussian window by any window function $\phi \in M^{1}$.

Gröchenig and Leinert GL04 proved that if $\Lambda$ is a lattice, then the canonical dual frame of a lattice Gabor frame generated by a function $g \in M^{1}$ is generated by a dual window that also lies in $M^{1}$ (they also obtained weighted versions of this result). Their proof relied on deep results about symmetric Banach algebras. The following result proved in BCHL06b holds in the general setting of irregular Gabor frame sequences. Note in particular that this result also applies to Gabor Riesz sequences.

Theorem 4.9. Let $g \in M^{1}$ and $\Lambda \subset \mathbf{R}^{2 d}$ be such that $\mathcal{G}(g, \Lambda)$ is a Gabor frame sequence in $L^{2}\left(\mathbf{R}^{d}\right)$, with canonical dual frame sequence $\tilde{\mathcal{G}}=\left\{\tilde{g}_{\lambda}\right\}_{\lambda \in \Lambda}$. Then the following statements hold:

(a) $\tilde{g}_{\lambda} \in M^{1}$ for all $\lambda \in \Lambda$,

(b) $\sup _{\lambda}\left\|\tilde{g}_{\lambda}\right\|_{M^{1}}<\infty$, and

(c) $\tilde{\mathcal{G}}$ is a set of Gabor molecules with respect to an envelope $\Gamma \in W\left(\mathcal{C}, \ell^{1}\right)$.

Furthermore, the same conclusions hold when $\tilde{\mathcal{G}}$ is replaced by the canonical Parseval frame $S^{-1 / 2}(\mathcal{G}(g, \Lambda))$.

\section{ACKNOWLEDGMENTS}

We gratefully acknowledge conversations with Karlheinz Gröchenig, Massimo Fornasier, Hans Feichtinger, Norbert Kaiblinger, Ilya Krishtal, Gitta Kutyniok, Henry Landau, and Thomas Strohmer. 


\section{REFERENCES}

[BCHL06a] R. Balan, P. G. Casazza, C. Heil, and Z. Landau, Density, overcompleteness, and localization of frames, I. Theory, J. Fourier Anal. Appl., 12 (2006), 105-143.

[BCHL06b] R. Balan, P. G. Casazza, C. Heil, and Z. Landau, Density, overcompleteness, and localization of frames, II. Gabor frames, J. Fourier Anal. Appl., 12 (2006), 307-344.

[Bas90] A. G. Baskakov, Wiener's theorem and asymptotic estimates for elements of inverse matrices, Funktsional. Anal. i Prilozhen., 24 (1990), 64-65; translation in Funct. Anal. Appl., 24 (1990), 222-224. MR.1082033 (92g:47049)

[CCLV05] P. G. Casazza, O. Christensen, A. Lindner, and R. Vershynin, Frames and the Feichtinger conjecture, Proc. Amer. Math. Soc., 133 (2005), 1025-1033. MR2117203 (2006a:46024)

[CT06] P. G. Casazza and J. C. Tremain, The Kadison-Singer problem in mathematics and engineering, Proc. Natl. Acad. Sci. USA, 103 (2006), 2032-2039. MR2204073

[Chr03] O. Christensen, An introduction to frames and Riesz bases, Birkhäuser, Boston, 2003.

[CDH99] O. Christensen, B. Deng, and C. Heil, Density of Gabor frames, Appl. Comput. Harmon. Anal., 7 (1999), 292-304. MR1721808 (2000j:42043)

[CFZ01] O. Christensen, S. Favier, and Z. Felipe, Irregular wavelet frames and Gabor frames, Approx. Theory Appl. (N.S.), 17 (2001), 90-101. MR.1885530(2003c:42033)

[Dau90] I. Daubechies, The wavelet transform, time-frequency localization and signal analysis, IEEE Trans. Inform. Theory, 39 (1990), 961-1005. MR1066587 (91e:42038)

[Dau92] I. Daubechies, Ten lectures on wavelets, SIAM, Philadelphia, 1992. MR.1162107 (93e:42045)

[DLL95] I. Daubechies, H. Landau, and Z. Landau, Gabor time-frequency lattices and the Wexler-Raz identity, J. Fourier Anal. Appl., 1 (1995), 437-478. MR1350701 (96i:42021)

[DS52] R. J. Duffin and A. C. Schaeffer, A class of nonharmonic Fourier series, Trans. Amer. Math. Soc., 72 (1952), 341-366. MR0047179 (13:839a)

[Fei80] H. G. Feichtinger, Banach convolution algebras of Wiener type, Functions, Series, Operators, Proc. Conf. Budapest 38, Colloq. Math. Soc. János Bolyai, 1980, pp. 509-524. MR0751019 (85j:43005)

[Fei81] H. G. Feichtinger, On a new Segal algebra, Monatsh. Math., 92 (1981), 269-289. MR 0643206 (83a:43002)

[FG89a] H. G. Feichtinger and K. Gröchenig, Banach spaces related to integrable group representations and their atomic decompositions, I, J. Funct. Anal., 86 (1989) 307-340. MR.1021139 (91g:43011)

[FG89b] H. G. Feichtinger and K. Gröchenig, Banach spaces related to integrable group representations and their atomic decompositions, II, Monatsh. Math., 108 (1989) 129-148. MR.1026614 (91g:43012)

[For03] M. Fornasier, Constructive methods for numerical applications in signal processing and homogenization problems, Ph.D. Thesis, U. Padua, 2003.

[GKW89] I. Gohberg, M. A. Kaashoek, and H. J. Woerdeman, The band method for positive and contractive extension problems, J. Operator Theory, 22 (1989), 109-155. MR 1026078 (91a:47021)

[GKK01] V. K. Goyal, J. Kovačević, and J. A. Kelner, Quantized frame expansions with erasures, Appl. Comput. Harmon. Anal., 10 (2001), 203-233. MR.1829801(2002h:94012)

[Grö01] K. Gröchenig, Foundations of time-frequency analysis, Birkhäuser, Boston, 2001. MR:1843717 (2002h:42001)

[Grö03] K. Gröchenig, Localized frames are finite unions of Riesz sequences, Adv. Comput. Math., 18 (2003), 149-157. MR.1968117 (2004a:42044)

[Grö04] K. Gröchenig, Localization of frames, Banach frames, and the invertibility of the frame operator, J. Fourier Anal. Appl., 10 (2004), 105-132. MR2054304 (2005f:42086)

[GF05] K. Gröchenig and M. Fornasier, Intrinsic localization of frames, Constr. Approx., 22 (2005), 395-415. MR2164142(2006f:42030)

[GL04] K. Gröchenig and M. Leinert, Wiener's lemma for twisted convolution and Gabor frames, J. Amer. Math. Soc., 17 (2004), 1-18. MR2015328 (2004m:42037) 
[GR96] K. Gröchenig and H. Razafinjatovo, On Landau's necessary density conditions for sampling and interpolation of band-limited functions, J. London Math. Soc. (2), 54 (1996), 557-565. MR1413898 (98m:42029)

[HW01] D. Han and Y. Wang, Lattice tiling and the Weyl-Heisenberg frames, Geom. Funct. Anal., 11 (2001), 742-758. MR.1866800 (2003j:52021)

[Hei03] C. Heil, An introduction to weighted Wiener amalgams, Wavelets and Their Applications (Chennai, January 2002), M. Krishna, R. Radha and S. Thangavelu, eds., Allied Publishers, New Delhi, 2003, pp. 183-216.

[Jan95] A. J. E. M. Janssen, Duality and biorthogonality for Weyl-Heisenberg frames, J. Fourier Anal. Appl., 1 (1995), 403-436. MR1350700(97e:42007)

[Jan98] A. J. E. M. Janssen, A density theorem for time-continuous filter banks, Signal and Image Representation in Combined Spaces, Y. Y. Zeevi and R. R. Coifman, eds., Wavelet Anal. Appl., Vol. 7, Academic Press, San Diego, CA, 1998, pp. 513-523. MR.1614987 (99d:42061)

[KR05] A. Klappenecker and M. Rötteler, Mutually unbiased bases, spherical designs, and frames, Wavelets XI (San Diego, CA, 2005), Proc. SPIE 5914, M. Papadakis et al., eds., SPIE, Bellingham, WA (2005), pp. 196-208.

[LW03] Y. Liu and Y. Wang, The uniformity of non-uniform Gabor bases, Adv. Comput. Math., 18 (2003), 345-355. MR.1968125 (2004h:42036)

[RS95] J. Ramanathan and T. Steger, Incompleteness of sparse coherent states, Appl. Comput. Harmon. Anal., 2 (1995) 148-153. MR1325536 (96b:81049)

[SHHS01] A. Shokrollahi, B. Hassibi, B. M. Hochwald, and W. Sweldens, Representation theory for high-rate multiple-antenna code design, IEEE Trans. Inform. Theory, 47 (2001), 2335-2367. MR1873925 (2003j:94120)

[Sjö95] J. Sjöstrand, Wiener type algebras of pseudodifferential operators, Séminaire sur les Équations aux Dérivées Partielles, 1994-1995, Exp. No. IV, École Polytech., Palaiseau, 1995. MR 1362552 (96j:47049)

[SH03] T. Strohmer and R. W. Heath, Jr., Grassmannian frames with applications to coding and communication, Appl. Comput. Harmon. Anal., 14 (2003), 257-275. MR.1984549 (2004d:42053)

[SZ02] W. Sun and X. Zhou, Irregular wavelet/Gabor frames, Appl. Comput. Harmon. Anal., 13 (2002), 63-76. MR.1930176 (2003i:42057)

[WGW04] P. J. Wolfe, S. J. Godsill, and W.-J. Ng, Bayesian variable selection and regularization for time-frequency surface estimation, J. R. Stat. Soc. Ser. B Stat. Methodol., 66 (2004), 575-589. MR2088291

[UAL03] M. Unser, A. Aldroubi, and A. Laine, eds., Special Issue on Wavelets in Medical Imaging, IEEE Trans. Medical Imaging, 22 (2003).

[You01] R. Young, An introduction to nonharmonic Fourier series, Revised first edition, Academic Press, San Diego, 2001. MR1836633(2002b:42001)

Siemens Corporate Research, 755 College Road East, Princeton, New Jersey 08540

E-mail address: radu.balan@siemens.com

Department of Mathematics, University of Missouri, Columbia, Missouri 65211

E-mail address: pete@math.missouri.edu

School of Mathematics, Georgia Institute of Technology, Atlanta, Georgia 30332

E-mail address: heil@math.gatech.edu

Department of Mathematics R8133, The City College of New York, Convent Avenue at 138th Street, New York, New York 10031

E-mail address: landau@sci.ccny.cuny.edu 